Research Paper

\title{
MiR-34c promotes hepatic stellate cell activation and Liver Fibrogenesis by suppressing ACSL1 expression
}

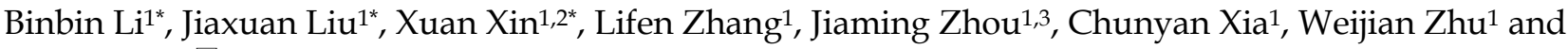 \\ Hongyu $\mathrm{Yu}^{1}$ \\ 1. Department of Pathology, Changzheng Hospital, Navy Medical University (Second Military Medical University), Shanghai 200003, China. \\ 2. Department of Pathology, No. 960 Hospital of People' Liberation Army, Jinan 250031, China. \\ 3. Department of Pathological Anatomy, Nantong University, Nantong 226001, China. \\ *These authors contributed equally to this work. \\ $\square$ Corresponding author: Hongyu Yu, Department of Pathology, Changzheng Hospital, Navy Medical University (Second Military Medical University), \\ Shanghai 200003, China. E-mail: yuhongyu795@smmu.edu.cn; Tel.: 00-86-021-81886121. \\ (c) The author(s). This is an open access article distributed under the terms of the Creative Commons Attribution License (https://creativecommons.org/licenses/by/4.0/). \\ See http://ivyspring.com/terms for full terms and conditions.
}

Received: 2020.08.05; Accepted: 2020.12.02; Published: 2021.01.01

\begin{abstract}
Normally, there are multiple microRNAs involved in the pathogenesis of liver fibrosis. In our work, we aimed at identifying the role of miR-34c in the hepatic stellate cell (HSC) activation and liver fibrosis and its potential mechanism. Our results have shown that during natural activation of HSC, the level of miR-34c was increased significantly whereas acyl-CoA synthetase long-chain family member-1(ACSL1), which is a key enzyme can affect fatty acid(FA) synthesis, was decreased. A double fluorescence reporter assay further confirmed that ACSLI is a direct target gene of miR-34c. Moreover, the inhibition of miR-34C can attenuate the synthesis of collagen in HSC-T6. In our rescue assay, ACSL1 expression was 1.49-fold higher compared to normal control cells which were transfected with the miR-34c inhibitor in a stable low expression ACSL1 cell line. While at the same time, $\alpha$-SMA and Coll $\alpha$ expression decreased by $18.22 \%$ and $2.58 \%$, respectively. Moreover, we performed an in vivo model using dimethylnitrosamine (DMN) in conjunction with the miR-34c agomir, combined with the treatment of DMN and the miR-34c agomir can increase liver fibrosis. Meanwhile, the degree of hepatic fibrosis was increased and lipid droplets reduced dramatically in rats and HSC-T6 cell treated with miR-34c mimics alone compared to untreated groups. Our results indicate that miR-34c plays an essential role in liver fibrosis by targeting ACSLI closely associated with lipid droplets, and it might be used as a potential therapeutic target.
\end{abstract}

Key words: miR-34c; ACSL1; liver fibrogenesis; fatty acid; lipid metabolism

\section{Introduction}

Liver disease is a major cause of death and illness around the world. Specifically, liver fibrosis is a common key pathology resulting from a variety of chronic liver diseases [1, 2]. If there is no suppression or reversal of liver fibrosis, fibrosis can progress to cirrhosis which is a critical risk factor for liver cancer and a challenge for human health, resulting in 1.16 million deaths annually worldwide [3]. However, no effective prevention and treatment are available due to mechanisms of liver disease have not been completely elucidated [2, 4, 5]. Among liver cells, Kupffer cells, endothelial cells, and hepatic stellate cells (HSC) are involved in the development of liver fibrosis. HSC activation and proliferation represent pivotal mechanisms that trigger liver fibrosis [6-8]. In recent years, microRNAs (miRNAs) have been recognized as one of the most important regulators in post-transcriptional regulation of gene expression and the differential expression of multiple miRNAs is related to HSC activation, proliferation and even apoptosis closely [9]. A growing body of research indicates that many miRNA families are associated with HSC activation and the development of liver fibrogenesis by synergistically modulating certain signaling pathways [10-13]. In our previous study, miRNAs were differentially expressed in animal 
models of liver fibrosis, as determined by chip screening. MiR-34 a/c expression is significantly up-regulated and positively correlates with the development of liver fibrosis [14]. The miR-34 family has three members, including miR-34a, miR-34b and miR-34c [15]. In mammals, miR-34a is located at chromosome 1 p36 while miR-34b and miR-34c share primary transcription from chromosome 11q23 [15, 16]. There are numerous reports of alterations in miR-34 expression in cancer highlighting the importance of this microRNA family in oncogenesis. However, the role of miR-34c in HSC activation and liver fibrosis as well as its potential mechanism is still unclear.

We have initially confirmed the miR-34 family targets acyl-CoA synthetase long-chain family member-1 ACSL1 [14]. Members of the ACSL family include five different ACSL isoforms. ACSL1, ACSL4 and ACSL5 are present in the liver and adipocytes, whereas only ACSL1 is a key enzyme with triglyceride synthesis [17-19]. In this study, we demonstrated the function and molecular mechanisms of miR-34c in hepatic fibrosis. Firstly, we isolated rat primary HSC and examined $\mathrm{miR}-34 \mathrm{c}$ and ACSL1 expression during natural activation of HSC. Our results showed that miR-34c was significantly up-regulated whereas ACSL1 was down-regulated. Then the double-luciferase reporter assay was used to confirm that ACSL1 was a direct target gene of miR-34c. Furthermore, inhibition of miR-34C attenuated expression of profibrotic markers and cell proliferation in HSC in vitro. These findings were subsequently verified in rescue assay. It has shown that miR-34c inhibition partly restores the effect of ACSL1 function.

In normal livers, HSC is quiescent and filled with cytoplasmic lipid droplets containing retinoids (especially retinyl palmitate) and triacylglycerols, accounting for more than $70 \%$ of lipids $[8,20]$. HSC transition from quiescent state to fibrosis, characterized by the gradual loss of lipid droplets and retinoids, is accompanied by increased a-smooth muscle actin (a-SMA) and type I collagen (Col1a) expression [21, 22]. Therefore, exploring potential factors affecting HSC activation and relationship between lipid and liver fibrosis is useful for elucidating potential molecular mechanisms. Animal experiments have further confirmed that miR-34c promotes liver fibrosis and surely lipid accumulation in the liver was significantly reduced while conducting animal experiments. Moreover, in vitro, transfected with miR-34c mimic and inhibitor in HSC-T6, the result was consistent with vivo data by Sudan black staining.

In general, our findings revealed that miR-34c plays an essential role in liver fibrosis by targeting ACSL1 closely associated with lipid, and it might be used as a potential therapeutic target.

\section{Materials and Methods}

\section{Cell culture and transfection}

HSCs were considered quiescent on day 2 and then activated on day 14 . The day before transfection, HSCs were seeded into 6-well plates and 2,000 $\mu \mathrm{l}$ of high-glucose dulbecco modified eagle medium (DMEM) containing 10\% fetal bovine serum (FBS) was added. Due to the fast growth of activated HSC, the cell density was $3 \times 10^{5}$. Cells were transfected with an inhibitor of miR-34c or with a control NC sequence using a riboMONITORTM transfection indicator. The rate of cell growth, cell status and contamination were studied 6 to $72 \mathrm{~h}$ after transfection.

\section{Immunofluorescence assay}

In a 6-well plate, the slides of the grafted cells were immersed in PBS three times for 3 min each cycle. Then, slides were fixed in formaldehyde solution for $10 \mathrm{~min}$, and washed with PBS three times for $1 \mathrm{~min}$ each, and treated with $0.5 \%$ Triton X-100 (formulated in PBS) for $20 \mathrm{~min}$ at room temperature. Slides were again rinsed with PBS three times for 3 min and treated with normal goat blocking serum at room temperature for $30 \mathrm{~min}$. Then primary antibodies were added (a-SMA, 1:100; Desmin 1:100) and slides were placed in a wet box and incubated overnight at $4^{\circ} \mathrm{C}$. The following day, slides were washed with PBS-Tween (PBS-T) three times for $3 \mathrm{~min}$ each. Finally, the fluorescent secondary antibody was added and the reaction was incubated in a wet box at $20-37^{\circ} \mathrm{C}$ for $1 \mathrm{~h}$. Slides were again washed with PBS-T, three times for $3 \mathrm{~min}$ each, and counterstained with DAPI for $5 \mathrm{~min}$. Microphotographs were taken and saved.

\section{RNA extraction and qRT-PCR analyses}

Total RNA was extracted using the Trizol reagent (Invitrogen, USA) according to the manufacturer's protocol, and total RNA was quantified using a Nanodrop 3300 (Thermo Scientific, Wilmington, DE). $500 \mathrm{ng}$ of RNA was reverse transcribed into cDNA according to the manufacturer's instructions (Takara, China) followed by amplification of cDNA using SYBR GREEN (Takara, China) with the assistance of the CFX96 Touch system (Bio-Rad, USA). ACSL1, Col1a and a-SMA and mature miR-34C relative expression levels were calculated using GADPH and U6 snRNA as housekeeping genes. The upstream and downstream primers required for qRT-PCR were designed and 
provided by Shanghai Biotech Co., Ltd. The primers used are showed in Table 1.

Table 1. Primer sequences

\begin{tabular}{ll}
\hline Gene (mouse) & Primer sequences $\left(5^{\prime}-3^{\prime}\right)$ \\
\hline ACSL1 & Forward: TGTCCATGGAGAAAGCTTG \\
& Reverse: GATATCCTTGTTCCTGCACAG \\
GAPDH & Forward: ACAGCAACAGGGTGGTGGAC \\
& Reverse: TTTGAGGGTGCAGCGAACTT \\
a-SMA & Forward: TTCCTTCGTGACTACTGCTGAG \\
& Reverse: CAATGAAAGATGGCTGGAAGAG \\
Col1a & Forward: ATGTCTGGTTTGGAGAGAGCA \\
& Reverse: GAGGAGCAGGGACTTCTTGAG \\
U6 & Forward: GCAGTGCTTAGCTGGTTGT \\
& Reverse: GCGAGCACAGAATTAATACGAC \\
miR-34c & Forward: CGCGGATCCTGCTGCGTGCTGTGATTC \\
& Reverse: GTGGAATTCTTTCCCTGTGGCTGTCCTC \\
\hline
\end{tabular}

\section{Western blotting}

The transfected cells were lysed in RIPA buffer (Biomed, China) to extract the total cellular protein. Protein concentration in the cell lysates was measured for protein using the BCA method. Depending on the size of the protein we used $5 \%$ or $10 \%(20-80 \mathrm{kD})$ SDS-PAGE separation gels. A total of 20ul of protein was loaded and proteins were separated by gel electrophoresis and transferred onto a PVDF membrane. The membrane was blocked in PBS-T with $5 \%$ non-fat dry milk for $1 \mathrm{~h}$ at room temperature. The protein bands were then incubated for $4 \mathrm{~h}$ at room temperature in anti-ACSL1 (Thermo), anti-Col1a rabbit polyclonal antibody (ab34710), anti- $\alpha$ SMA (ab5694). Anti-GAPDH rabbit monoclonal antibody (ab9485) was used as an internal control. Afterwards, the membranes were incubated in PBS-T diluted rabbit secondary antibody (1:5000) (Abmart, China) for $1 \mathrm{~h}$ at room temperature. Protein bands were visualized using ECL luminescent solution and the images of protein bands were got using a BIORAD Flour-S Muiltilmager Gel Imager.

\section{Luciferase reporter assay}

pMIR-REPORT-ACSL1-3'UTR wild-type plasmids (pMIR-ACSL1-WT), pMIR-REPORT-ACSL1 mutant plasmids (pMIR-ACSL1-MUT), pMIR-REPORT luciferase empty plasmids (pMIR) and plasmid containing overexpression of miR-34c (miR-34c up) or control miRNA (miR-NC up) (purchased from Shanghai Jikai Gene Chem Co., Ltd.) were co-transfected into HEK293, and the empty vector was used as control. Cells were seeded in 24-well plates before transfection, and the density of each well was about $3 \times 10^{5}$. Fluorescent gene expression in the plasmid was observed to determine transfection efficiency after $24 \mathrm{~h}$. The fluorescence of Firefly and Renilla luciferase were detected using a microplate reader.

\section{Construction and screening of stable HSC lines with low expression of ACSLI}

According to the RNAi sequence design principle, three pairs of shRNA oligonucleotide sequences targeting the ACSL1 mRNA target sequence were designed: shR1 (GCGATAATCTGTTT CACAAGT), shR2 (GGATGACCTCAAGGTGCT TCA), and shR3 (G CCCTAGATAAAGATGGTTGG). shRCON (TTCTCCGAACGTGTCACGT) lacking homology to any known human sequence was used as a control parameter. Synthesis of the shRNA DNA oligonucleotides, annealed to form double-stranded. The recombinant plasmids were cloned into plasmid pLKD-CMV-G \& PR-U6 by restriction endonucleases Age I and EcoR I and transformed into E. coli DH5a. The recombinant positive clones were identified by restriction enzyme digestion and sequenced. The recombinant lentiviral expression vector and lentivirus packaging plasmid were co-transfected into $293 \mathrm{~T}$ cells (named as Lenti-ACSL1-shRNA) to obtain the lentivirus concentrate, and the virus titer was calculated. HSC-T6 cells were infected with lentiviral particles of the appropriate titer $(\mathrm{MOI}=200)$ in stable cell line screening experiments. Cells that had not been effectively infected were killed by adding and maintaining $2 \mathrm{ug} / \mathrm{ml}$ of puromycin, and stably mixed stable strains were screened after 14 days. Real-time fluorescence quantitative PCR (RT-qPCR) was used to detect the change of ACSL1 gene expression in HSC-T6 cells, and the shRNA sequence with the best interference effect was screened out for further experiments.

\section{Animal experiments}

Three-week-old Sprague-Dawley rats, weighing $50 \pm 5 \mathrm{~g}$, were obtained from the Institute of Zoology, Chinese Academy of Sciences. All rats had free access to standard laboratory food and water in a pathogen free animal housing facility. Twenty-eight rats were randomly divided into three groups and labeled with picric acid. The model group (\#1 - \#12) was only challenged with $0.1 \% \mathrm{DMN}$ (10 mg/ $\mathrm{kg}$ body weight, intraperitoneal (i.p.) three times per week); model group + miR-34c agomir administration group (\#13 \#24), and the untreated control group (\#25 - \#28); The miR-34c agomir was dissolved in $100 \mu \mathrm{l}$ of saline and then injected into the tail vein at a dose of $100 \mu \mathrm{l} / 50 \mathrm{~g}$ body weight every $72 \mathrm{~h}$. Body weight was measured prior to adding each dose. Daily observation of rat activity and eating status was performed. Every other week, rats were randomly selected and sacrificed and the liver was extracted, weighed and fixed in formalin. Masson's trichrome and Sudan black 
staining was used to determine collagen and fat content, respectively. Four rats weighing about $50 \mathrm{~g}$ each were labeled with picric acid. One was used as a control group and three were injected with the miR-34c agomir. Surgery was performed under anesthesia minimizing animal pain, according to the criteria of the Committee of Ethics of our Institution. The research protocol was approved by the local Institutional Review Board to which the author belongs and was tested in accordance with the procedures and guidelines developed by the Animal Care and Use Committee of the Navy Medical University (Second Military Medical University), Shanghai, China.

\section{Masson's trichrome staining}

Rat liver paraffin sections were dewaxed with tap and distilled water, and then stained with Regaud hematoxylin dye or Weigert hematoxylin for 5-10 min. The slides were stained with Masson Ponceau Acidic Red for 5-10 min. Then the slides were placed into $2 \%$ acetic acid dissolved in aqueous solution and later in $1 \%$ phosphomolybdic acid aqueous solution for 3-5 min. Afterwards, without washing, sections were counterstained with aniline blue or green dye for 5 min followed by $0.2 \%$ acetic acid aqueous. Finally, the dehydration step was performed with alcohol and xylene, and coverslips were sealed with gum.

\section{Sudan black staining}

Fresh rat liver frozen 6-8 $\mu \mathrm{m}$ sections were cut and rinsed in $50 \%-70 \%$ alcohol for $20-30$ s. Sections were then stained using Sudan Black B dye for 3-5 min, and again rinsed in 50\%-70\% alcohol for a few seconds and then washed with distilled water. Nuclei were counterstained with red dye solution for $5 \mathrm{~min}$, washed for 1-2 $\mathrm{min}$, and covered with glycerol gelatin.

\section{Statistical Analysis}

SPSS v23.0 statistical software was used to analyze the results. Homogeneity of variance using one-way ANOVA (one-way ANOVA) was tested between groups (using LSD-T test). Kruskal-Wallis test was used when variance was not homogeneous; meanwhile values of each group were compared with each other by Nemenyi test. Statistical significance was considered when $p<0.05$. Graphpad Prism v6.01 was used to draw charts.

\section{Results}

MiR-34c is significantly up-regulated but ACSL1 is down-regulated during natural activation of HSC

MiR-34c and ACSL1 expression were evaluated in primary HSC on days 2, 7 and 14 . The experiment was repeated three times. Our results confirmed that the relative expression of miR-34c increased 47.6-fold in the 14-day treated HSC compared to quiescent HSC ( 2 days) $(P<0.001)$. Compared to the 7 day semi-activated HSC, the relative expression level of miR-34c in activated HSC was also dramatically different from that of quiescent and semi-activated HSC. Next, we measured the relative expression level of ACSL1 in semi-activated and activated HSC, which were significantly different from that of resting HSC $(P<0.001)$. The reduced ACSL1 expression was 98.1\% in activated HSC relative to quiescent HSC (Fig 1A). Based on these results, these experiments show that ACSL1 expression was significantly reduced throughout HSC activation.

Additionally, we performed immunofluorescence to identify the status of HSC activation in culture using a-SMA and Desmin antibodies. The results showed that 2 day-cultured HSC (quiescent) are a-SMA negative in the cytoplasm, but Desmin positive in the cytoplasm. However, a-SMA and Desmin expression were expressed in the cytoplasm of cultured HSC for 14 days (Fig. 1B).

\section{A double fluorescence reporter assay: ACSLI is a direct target gene of miR-34c}

To determine whether ACSL1 is a target gene of miR-34c or not, we divided our cells into six groups. Each group was transfected with: pMIR+ miR-NC up, pMIR+ miR-34c up, pMIR-ACSL1-WT + miR-NC up; pMIR+ACSL1-WT + miR-34c up, pMIR-ACSL1-MUT + miR-NC up or pMIR-ACSL1-MUT + miR-34c up. There was no significant difference in the fluorescence intensity between group pMIR+ miR-NC up and pMIR+ miR-34c up $(P=0.781)$ (Fig. 1C). However, when the ACSL1 wild-type plasmid was used to combine with the binding site of miR-34c (pMIR+ACSL1-WT + miR-34c up) the fluorescence intensity was significantly different from that of experimental group pMIR-ACSL1-WT +miR-NC up $(P<0.001)$ (Fig. 1D). No significant difference was found between pMIR-ACSL1-MUT + miR-NC up and pMIR-ACSL1-MUT + miR-34c up $(P=0.952)$ (Fig. 1E). Conclusively, these results suggest that ACSL1 is a direct target of miR-34c.

\section{Inhibition of miR-34C attenuates collagen synthesis and fibrogenesis in HSC-T6 cell line}

Experiments were carried out in the HSC-T6 HSC cell line. The experimental design consisted of three groups: the blank group - untreated HSC-T6 cells; the control group - HSC-T6 cells transfected with miRNA inhibitor Negative Control; and the group of HSC-T6 cells transfected with the miR-34c inhibitor. 
This experiment was repeated three times. $48 \mathrm{~h}$ after transfection, the results of qRT-PCR showed that miR-34c inhibition significantly reduced miR-34c expression in the experimental group compared to the blank and control groups (Fig. 2A). Conversely, ACSL1 expression was higher compared to the blank and control groups (Fig. 2A, B). We measured protein expression of ACSL1-target genes, including a-SMA and Colla using qRT-PCR and western blot analysis. In the miR-34c inhibition group, a-SMA and Col1a expression were significantly lower compared to the blank and control groups. (Fig. 2C, D). Our data indicate that inhibition of miR-34c attenuates the synthesis of collagen fibers in HSC cells.

\section{MiR-34c regulates ACSL1 expression and promotes initiation and development of liver fibrosis}

The $\mathrm{ccd} B$ virulence genes downstream of the U6 promoter of vector pLKD-CMV-G \& PR-U6-shRNA (Fig. 3A) were digested with Age I and EcoR I and inserted into the ACSL1 interference fragment to construct a plasmid that expresses green fluorescent protein EGFP and puromycin Rat ACSL1-resistant vector. qRT-PCR was used to detect mRNA expression of ACSL1 in three different interfering strains to understand the interference efficiency. The results showed that mRNA expression of ACSL1 in shR1, shR 2 and shR3 decreased by $58 \%, 57 \%$ and $34 \%$,
A
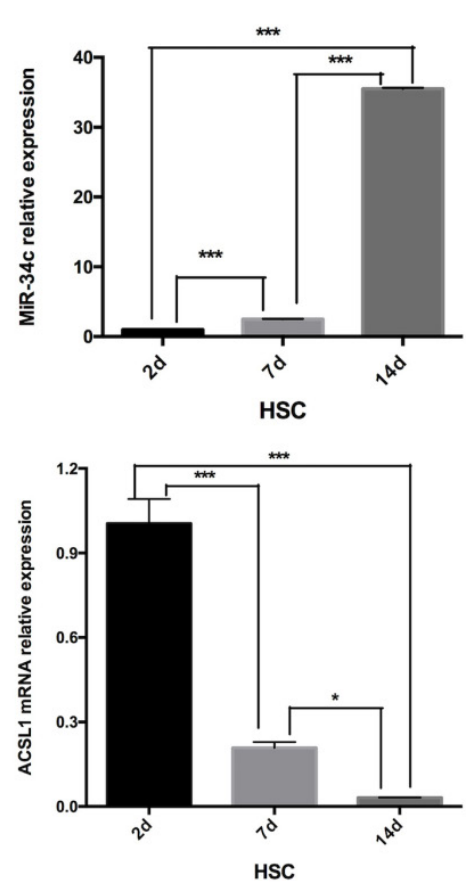

C

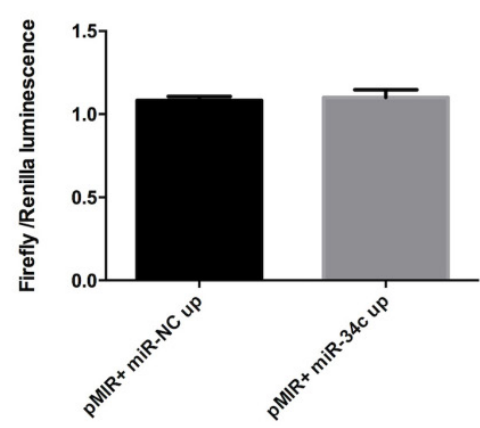

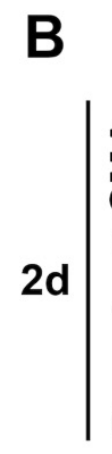
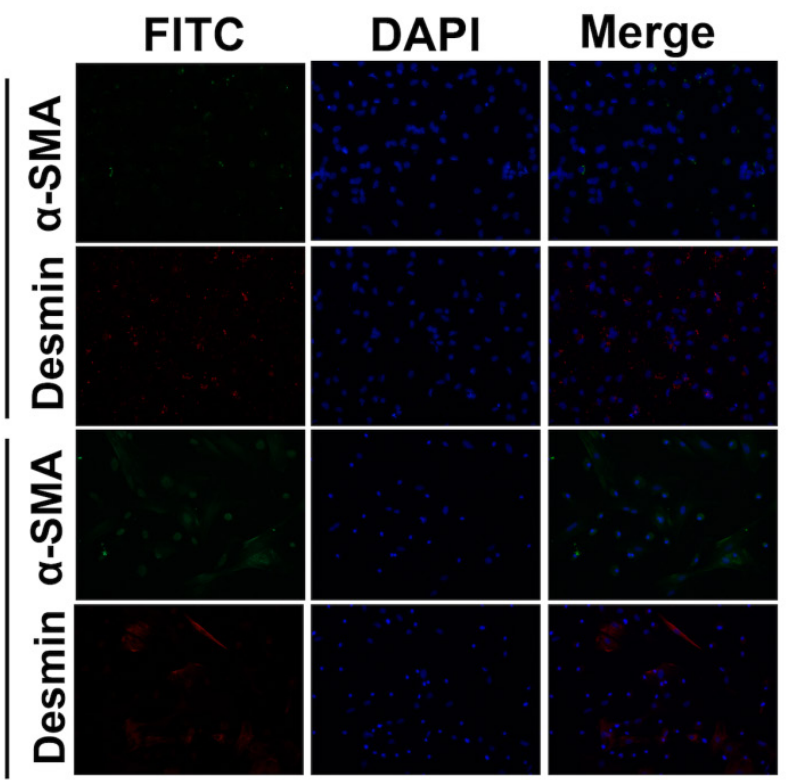

E
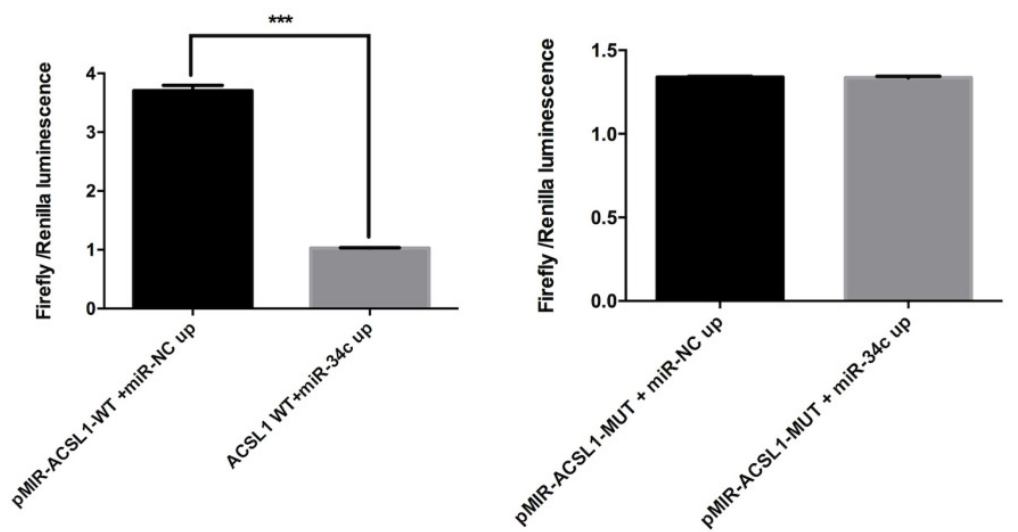

Figure 1. miR-34c up-regulation and ACSLI down-regulation are associated with HSC activation. (A) The relative expression of miR-34c increased 47.6-fold in 14-day (activated) HSC compared to 2-day (stationary) HSC $(P<0.001)$. The relative expression of ACSL1 decreased 0.98 -fold $(P<0.001)$ compared to the 2-day (stationary) HSC and 14-day (activated) HSC. (B) $\alpha$-SMA and Desmin staining was primarily evident in HSC under different states of immunofluorescence. (C)There was no significant difference in the fluorescence intensity between the experimental group ( $P M I R+$ miR-34c up) and the control group (PMIR+ miR-NC up) in cells overexpressing miR-34c $(P=0.781)$. (D) After modifying the binding site of miR-34c in the vector using the ACSL1 wild type plasmid in group PMIR+ACSLI-WT + miR-34c up, the fluorescence intensity in group PMIR+ACSL1-WT + miR-NC up was significantly different compared to the PMIR+ miR-NC up group $(P<0.001)$. (E) miR-34c could not bind to the mutant ACSL1 plasmid. There was no significant difference in fluorescence intensity between PMIR-ACSLI-MUT + miR-NC up and PMIR-ACSL1-MUT + miR-34c up $(P=0.952)$. 
respectively, compared to the control group. The $P$ values were all $<0.05$ and the differences were statistically significant (Fig. 3C). The results showed that shR1 interference stable cell line ACSL1 had the highest efficiency of interference. In the multiplicity of infection (MOI) of 200 conditions, HSC-T6 infection efficiency was approximately $80 \%$. After 14 days of puromycin drug screening, the rate of HSC-T6 expressing GFP was beyond $80 \%$ and the fluorescence brightness was enhanced (Fig 3B). This indicates that the stable expression of HSC-T6 cell line pLKD-CMVPuro-CMV-G\&PR-ACSL1 (shR1) was successful. In this rescue experiment, ACSL1 protein expression was 1.49-fold higher compared to the shR1 group after transfection with the miR-34c inhibitor in the constructed low-expression ACSL1 stable cell line (Fig. 3C). Concomitantly, the expression of a-SMA and Col1a, markers of hepatic fibrosis, decreased by $18.22 \%$ and $2.58 \%$, respectively (Fig. 3D). MiR-34c inhibition partly restored the function of ACSL1, further validating that $\mathrm{miR}-34 \mathrm{c}$ and promoting initiation and development of liver fibrosis by targeting ACSL1.

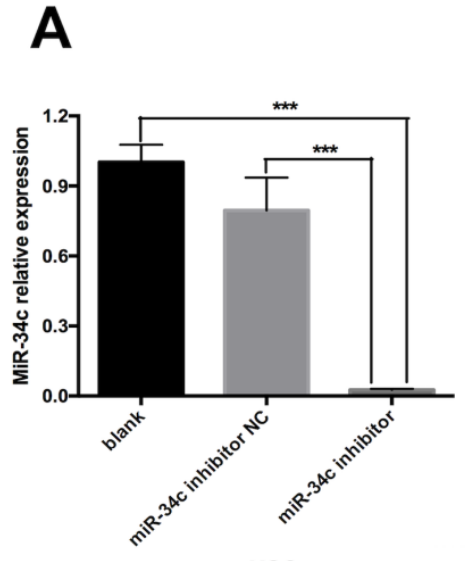

aHSC

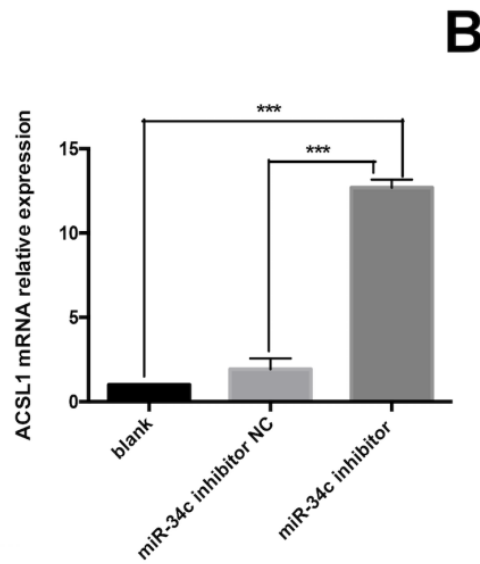

aHSC

B

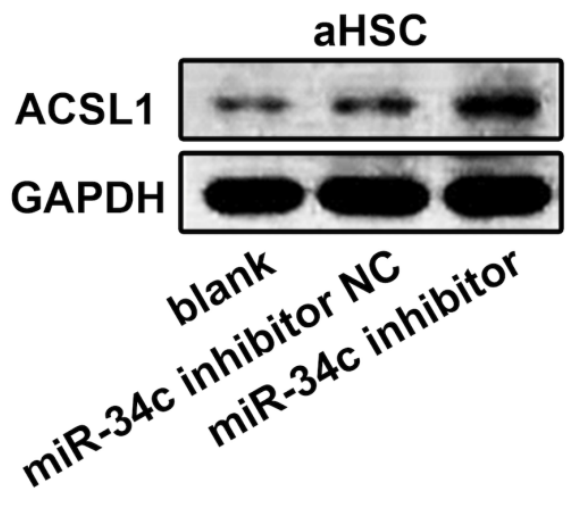

C

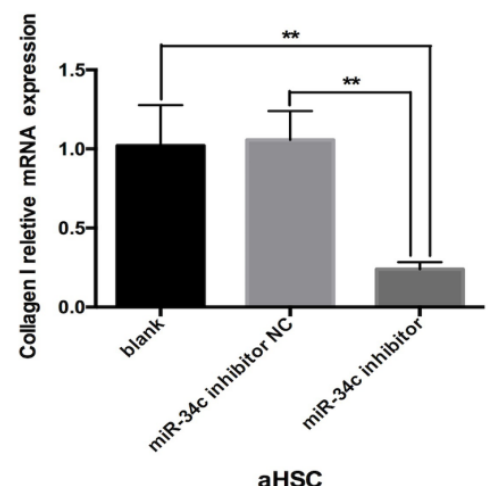

D
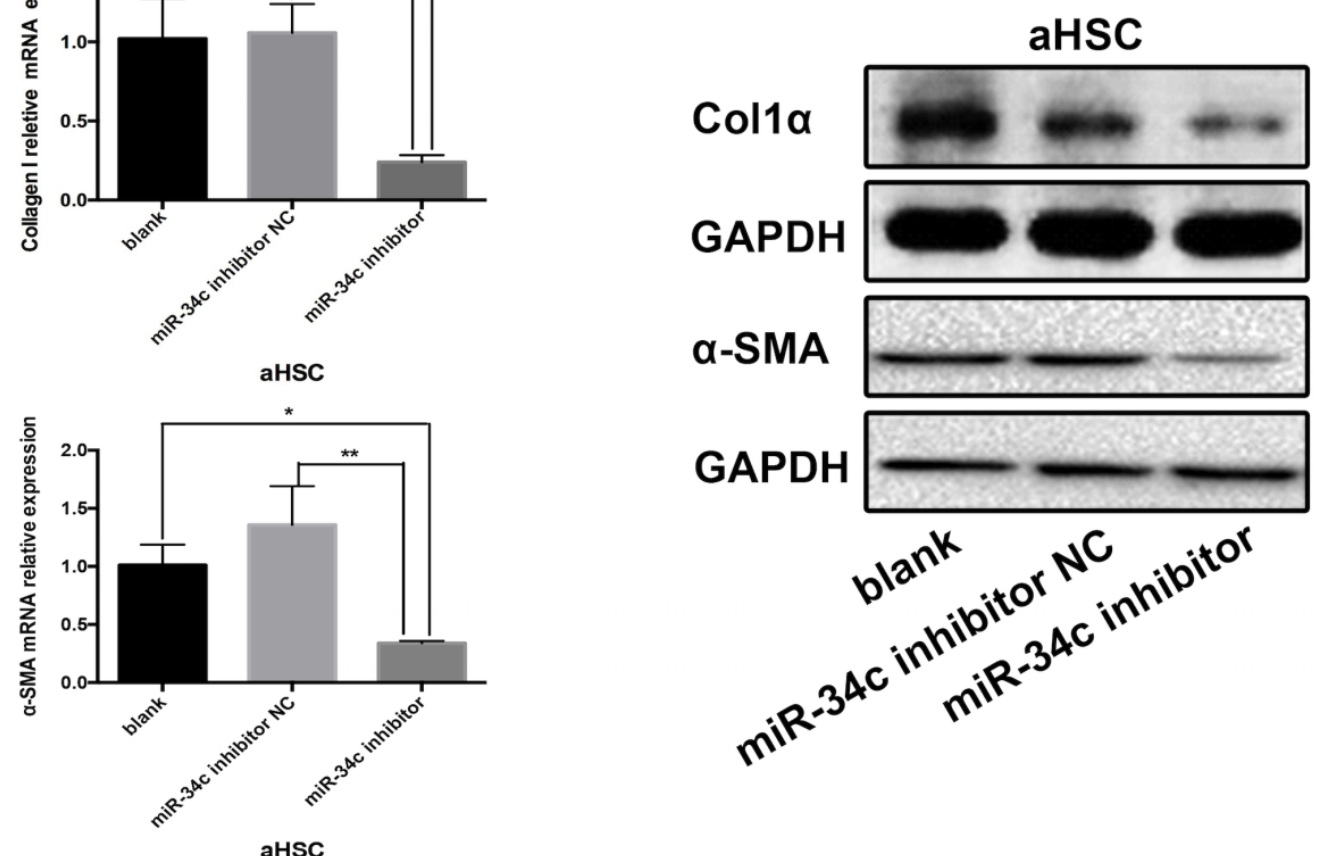

APDH 
A

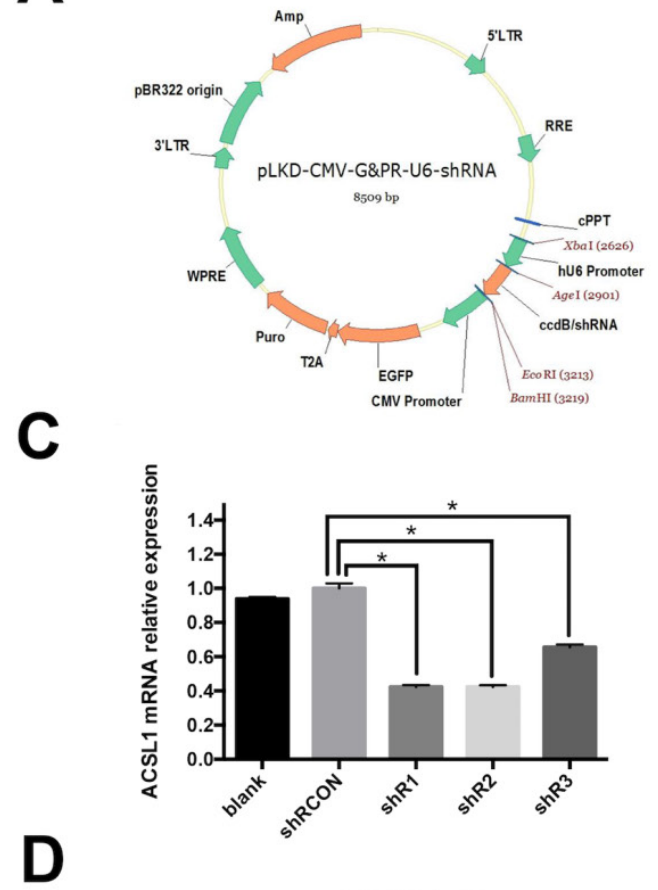

C

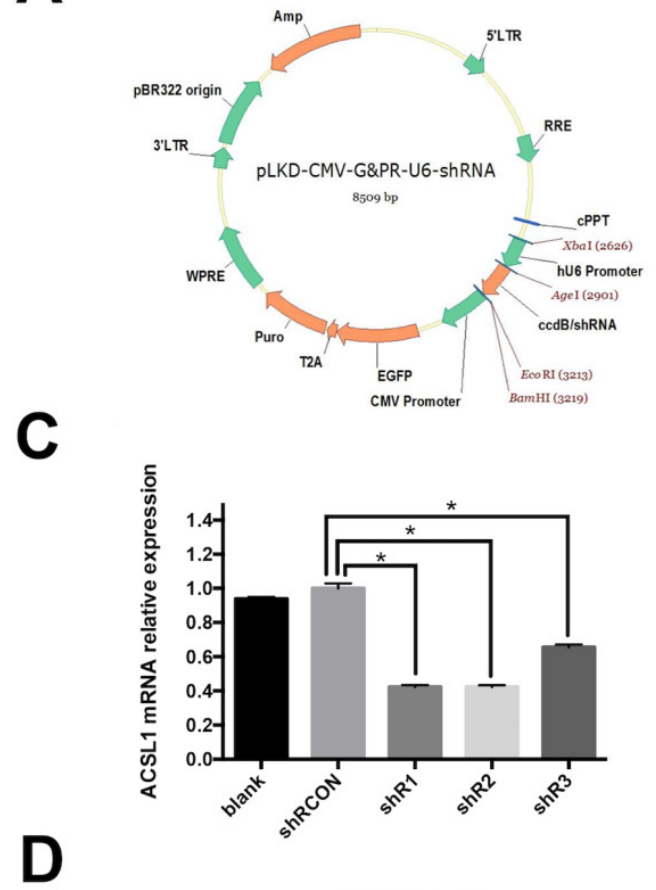

D
B

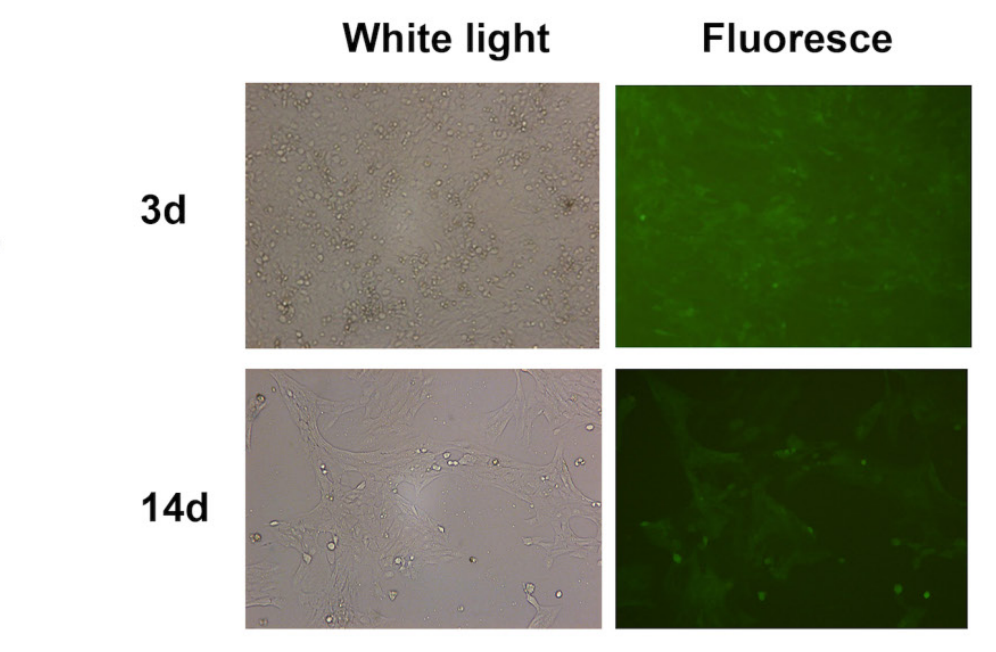

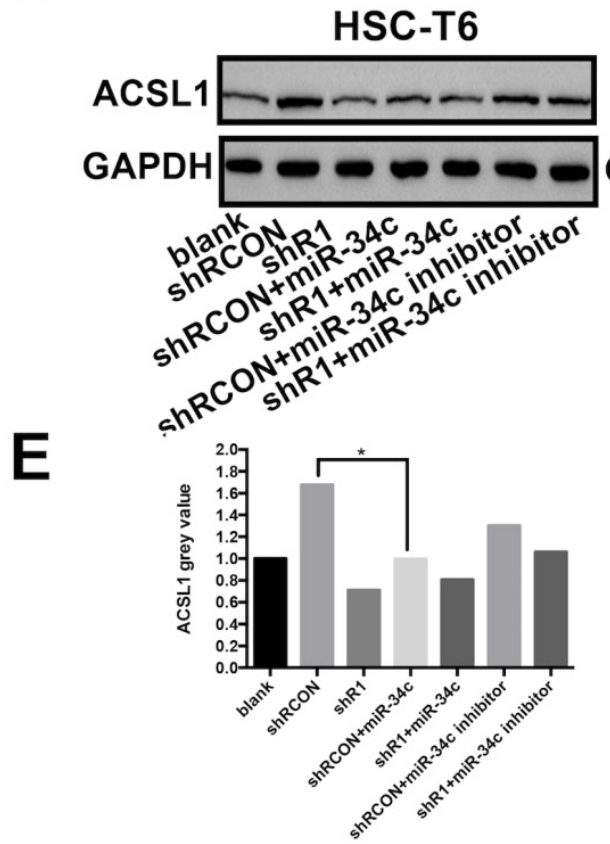
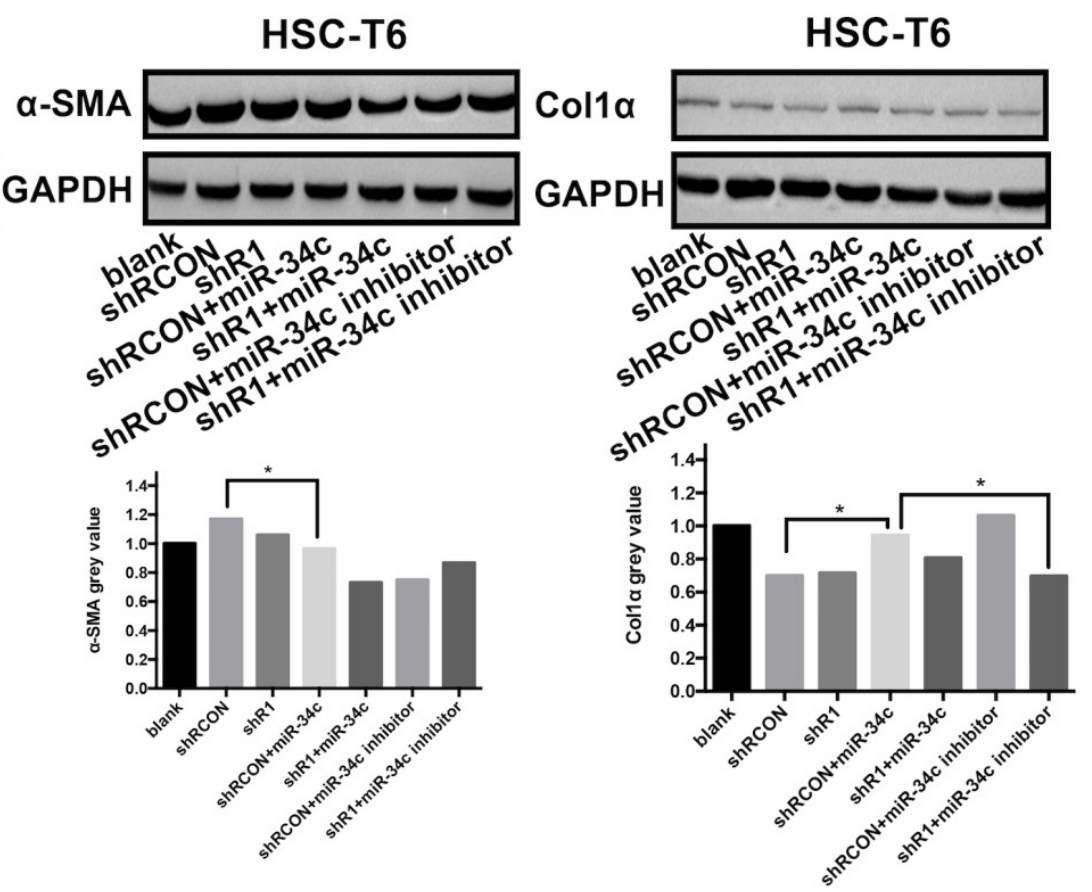

Figure 3. MiR-34c activates and proliferates HSC by targeting ACSLI. (A) Selected interference vectors pLKD-CMV-G\&PR-U6-shRNA vector map. (B) Lentivirus infected HSC-T6 cells, screened for fluorescence of stable strains after 3 and 14 days. (C) ACSL1 mRNA expression in each group was reduced by: $57.76 \%$, shR1 $(P<0.05)$; shR2 decreased by $57.67 \%(P<0.05)$; and shR3 decreased by $34.49 \%(P<0.05)$. (D) ACSLI, a-SMA, and Colla protein expression in each group. The internal reference is GAPDH. (E) Gray scale analysis of the automated chemiluminescence imaging system indicated that the expression of ACSL1 in the shR1+miR-34c inhibitor group increased 1.49 -fold, and the expression of $\alpha-S M A$ decreased by $18.22 \%$ and the expression of Colla decreased by $2.58 \%$.

MiR-34c promotes hepatic fibrosis and decrease liver lipid accumulation in rats and HSC-T6 cell line

In the current study, we established a model of early liver fibrosis using DMN in rats. Compared with the DMN model group, the degree of hepatic fibrosis and collagen fiber accumulation and deposition increased in rats treated with $\mathrm{DMN}$ and miR-34c agomir (Fig. 4A). In addition, Sudan black staining shows that the amount of lipid droplets in the miR-34c agomir group decreased compared to the blank control group (Fig. 4B). Moreover, we transfected miR-34c mimic and inhibitor in HSC-T6 cells. By Sudan black staining method, we found that lipid droplets were decreased in miR-34c mimic 
group. Conversely, lipid droplets were increased in miR-34c inhibitor group (Fig. 5A).

\section{Discussion}

Recently, there are some studies have demonstrated that abnormal expression of miRNAs in liver tissue are related to the pathogenesis of liver disease, including viral hepatitis, liver fibrosis, and hepatocellular carcinoma (HCC) [23-26]. Moreover, alteration of miRNAs target genes involved in hepatic energy metabolism, inflammatory, cell regeneration and fibrogenic signaling that drive the progression of nonalcoholic fatty liver disease (NAFLD) to liver fibrosis [27]. However, the mechanisms coordinating the interaction between the different signaling pathways of miRNAs regulating HSC activation and hepatic fibrosis have not been investigated in great detail.

A

Masson

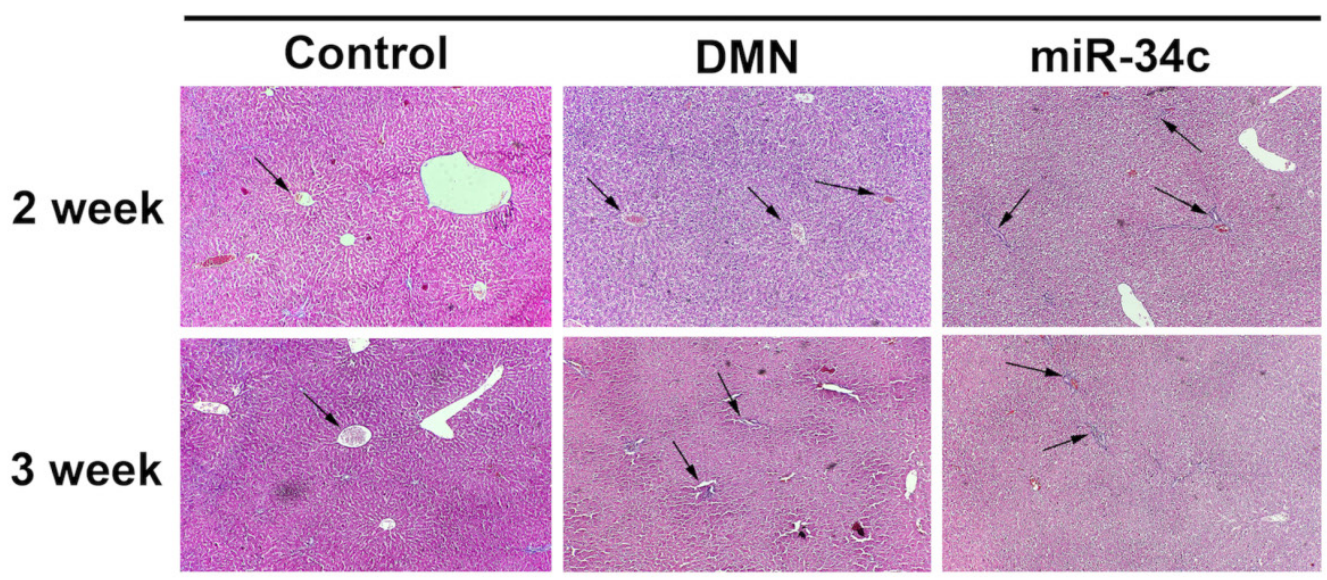

B

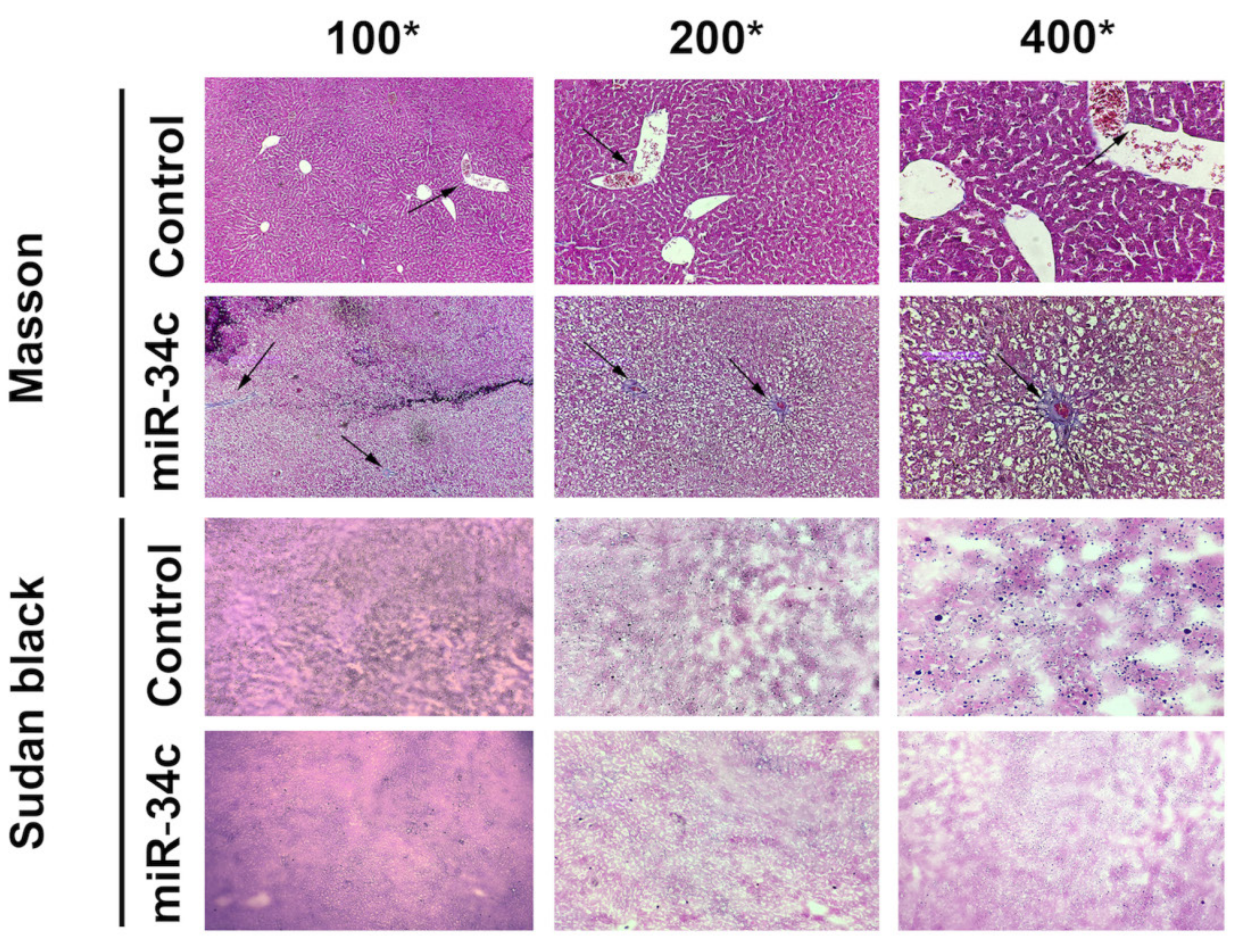

Figure 4. MiR-34c agomir promotes hepatic fibrosis and inhibits liver lipogenesis in rats. (A) Liver Masson trichrome staining in the normal control group, DMN model group, DMN model group, and the administration group was $100 \times$ for 2 weeks and 3 weeks; arrows point to the liver fibrosis areas. (B) Hepatic Masson trichrome stain and Sudan black stain in the DMN model group + administration group and normal control group at 4 weeks after administration. 100×, 200×, 400×, arrows indicate liver fibrosis areas. 


\section{Sudan black}

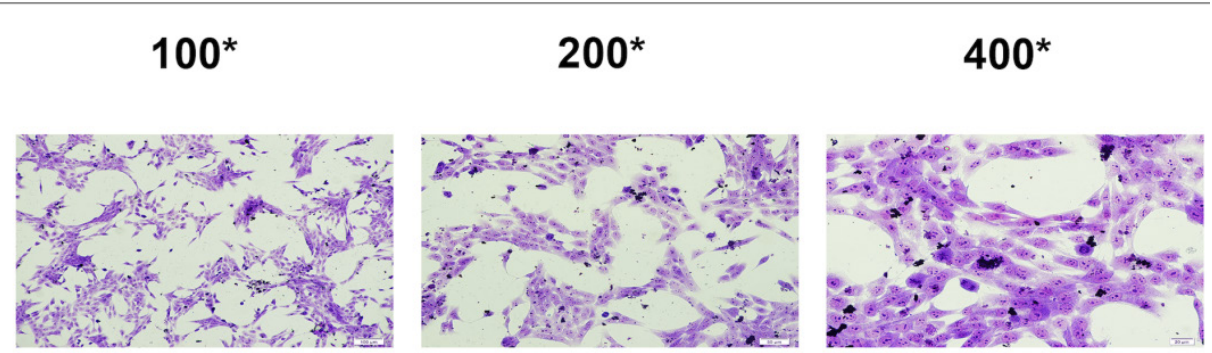

mimicNC
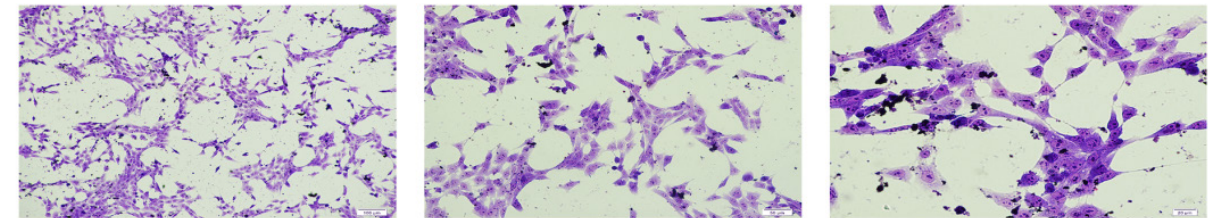

mimic
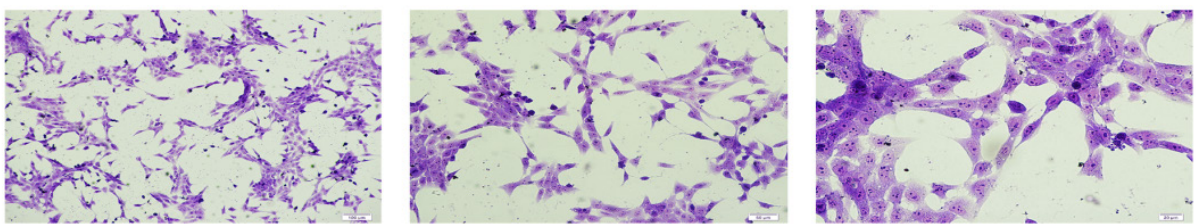

inhibitorNC
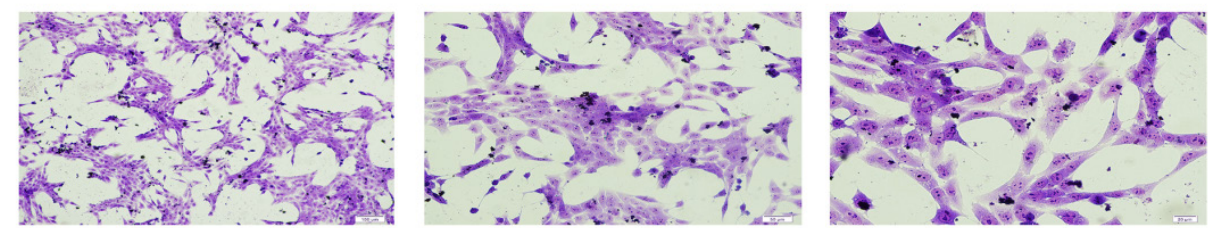

inhibitor
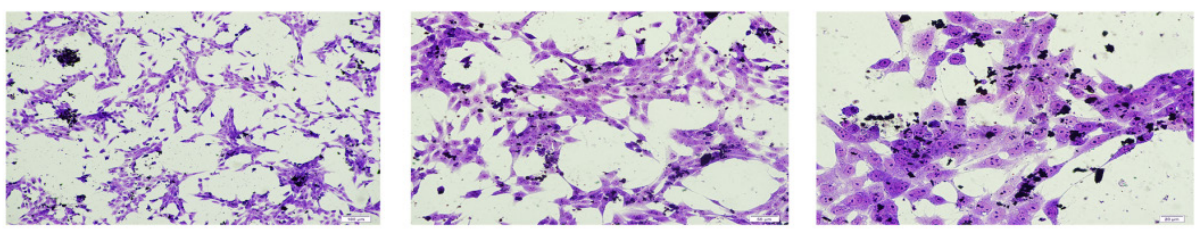

Figure 5. The content of lipid changes after transfected with MiR-34c mimic and inhibitor. HSC-T6 Sudan black staining in the blank group, normal control group, miR-34c mimic and inhibitor group, brownish black or dark black deposits are lipids, 100×, 200×, 400×.

In the current study, we explored whether miR-34c can directly targets ACSL1 or not and further investigated its role in HSC activation and hepatic fibrogenesis. First, we successfully extracted and cultured HSC. The expression of miR-34c was significantly increased during the natural activation of HSC, while ACSL1 was significantly decreased. This is consistent with our previous study in an animal model of liver fibrosis which showed that miR-34c expression increased along with liver fibrosis. Next, we confirmed that ACSL1 is indeed a direct target of miR-34c using a double fluorescent reporter system. We also found inhibition of miR-34c attenuates collagen synthesis in HSC_T6 cell line. By inhibiting miR-34c function in HSC, ACSL1 expression was significantly increased. This mechanism was associated with attenuated HSC activation and decreased hepatic fibrosis. Next, we used an inhibitor of miR-34c in a stable low expression ACSL1 cell line. The expression of ACSL1 at the protein level increased 1.49-fold, whereas the expression of a-SMA and Col1a in liver fibrosis decreased by $18.22 \%$ and $2.58 \%$, respectively. Our results showed that miR-34c inhibition partly restored the function of ACSL1, further validating that miR-34c targets ACSL1, thus regulating HSC activation and hepatic fibrogenesis. ACSL on the endoplasmic reticulum and mitochondrial outer membrane catalyzes the formation of acyl-CoA with FA of 12-20 carbon atoms, which are lipid metabolism intermediates and are involved in FA metabolism, membrane, modification, and several physiological processes [28, 29]. Besides, ACSL1 is critical in hepatic lipid homeostasis and is considered to play an essential role in activating FA synthesis of triglyceride (TG) $[30,31]$. These indicate that ACSL1 is closely related to FA and lipids. It has 
been reported that saturated FA, such as oleic acid (OA) and palmitic acid (PA), also participate in myofibroblast and HSC activation [21, 32].

To further investigate the regulation of miR-34c to hepatic fibrosis and the variety of lipid in vivo, we developed a rat model of early liver fibrosis using DMN models. Compared to the DMN model group, the degree of hepatic fibrosis increased in animals treated with a combination of DMN and miR-34c agomir, indicating that miR-34c promotes hepatic fibrogenesis which was in accordance with the results in activated HSC. Our findings suggested that miR-34c might play a promoting role in liver fibrogenesis, and its overexpression might be associated with the HSC activation. Moreover, we found a phenomenon that Sudan black staining revealed that the number of lipid droplets in the liver decreased in the miR-34c agomir group. However, so far, how miR-34c affects intrahepatic lipid content and thus affects liver fibrosis remains elusive. The liver is made up of many cell types [33]. But we don't know that lipid reduction in the liver occurs in which kind of cell. To further verify whether lipid droplets were associated with hepatic stellate cells or not which are closely related to liver fibrosis, we validated in a rat hepatic stellate cell line (HSC-T6). After transfected with miR-34c mimic and inhibitor, the result was consistent with vivo data by Sudan black staining. In all, we found out that miR-34c agomir can promote liver fibrosis and reduce lipid depositon in the liver. Both animal experiments and cell experiments verified miR-34c could affect liver fibrosis and lipid of hepatocytes and HSC.

In the liver, sustained TG and its hydrolysate FA, accretion leads to NAFLD, eventually progressing to NASH and cirrhosis [34]. More and more reports that a minority of NAFLD patients can lead to progressive nonalcoholic steatohepatitis (NASH), fibrosis, and ultimately HCC [35-37]. Our research has shown that miR-34c activates and proliferates HSC to further promote liver fibrosis by targeting ACSL1. Currently, $\mathrm{Li}$ and colleagues found that the miR-34 family participates in the process of hepatic fibrosis by regulating the Peroxisome proliferator-activated receptor $\gamma$ (PPAR $\gamma$ ) pathway [38]. PPAR- $\gamma$ and PPAR- $\alpha$ can regulate ACSL1 and increase the expression of genes involved in free FA formation and TG levels [31, 39, 40]. Another study shows that miR-34a can further regulate hepatic lipid metabolism by directly targeting PPARa signaling [41]. When we injected the 34c agomir, ACSL1 was significantly inhibited in vivo resulting in lipid content in the liver is affected and the FA synthesis of hepatocytes in the liver was changed, which significantly reduced the lipid droplets in whole liver by Sudan black staining.
The cellular lipidtartalom was reduced of miR-34c mimic group and was accumulated of miR-34c inhibitor group, which may be due to the fact that the miR-34c agomir suppressed the expression of ACSL1 and thus affected lipid synthesis in the liver. The process of our findings may be achieved by miR-34c targeting ACSL1 and altered regulation of the PPARY pathway. Given the accumulation of lipid in the liver, it was expected to play an important role in HSC activation and hepatic fibrosis. Therefore, it is necessary to further study the regulation of ACSL1 to lipid and the role of lipid in the development of hepatic fibrosis, it is of utmost interest to find new breakthroughs in the mechanisms of liver fibrogenesis and its reversal.

In summary, using functional experiments with HSC and in vivo experiments in rats, we further confirmed that miR-34c promotes development of hepatic fibrosis by targeting ACSL1. When miR-34c function was inhibited, HSC activation was inhibited, ACSL1 expression increased, and a-SMA and Col1a expression decreased. Our data confirmed that injection of the miR-34c agomir increased the degree of fibrosis but strongly attenuated lipidtartalom. Therefore, we hypothesize that the lipid metabolism pathway may be regulated by miR-34c inhibition, thus improving the course of liver fibrosis. Our results open a new therapeutic avenue/offer a promising way for the prevention and efficacious treatment strategies for hepatic fibrosis by investigating how miRNAs are involved in HSC activation and reversing hepatic fibrosis.

\section{Abbreviations}

HSC: hepatic stellate cells; ACSL1: acyl-CoA synthetase long-chain family member-1; FA: fatty acid; DMN: dimethylnitrosamine; miRNAs: microRNAs; a-SMA: a-smooth muscle actin; Col1a: type I collagen; DMEM: dulbecco modified eagle medium; FBS: fetal bovine serum; HCC: hepatocellular carcinoma; NAFLD: nonalcoholic fatty liver disease; TG: triglyceride; OA: oleic acid; PA: palmitic acid; NASH: nonalcoholic steatohepatitis; PPARY: peroxisome proliferator-activated receptor $\gamma$.

\section{Acknowledgements}

\section{Funding}

This study was supported by National Natural Science Foundation of China $(81370553,81870418)$.

\section{Author contributions}

BB. L, JX. L and X.X did the research work. BB. L and JX. L wrote this paper; $X . X$ did analysis of data; JX. L, BB. L, LF. Z and JM Zhou performed the study 
and interpreted the results of this study; CY. X edited and revised manuscript; WJ. $Z$ performed experiments. HY. Y supervised the study; HY. Y contributed to the design of this study and revising this paper.

\section{Innovation \& Noteworthy}

This is the first research showing miR-34c to have a regulatory effect on HSC activation and promotes hepatic fibrosis by targeting ACSL1, a key enzyme related to fatty acid and lipid synthesis closely. MiR-34c increased the degree of fibrosis but attenuated lipid accumulation. As a new target of gene therapy, miRNA is gradually showing its potential clinical application, our results open a new therapeutic avenue for the prevention and treatment of hepatic and a method of reversing hepatic fibrosis.

\section{Competing Interests}

The authors have declared that no competing interest exists.

\section{References}

1. Asrani SK, Devarbhavi H, Eaton J, Kamath PS. Burden of liver diseases in the world. J Hepatol. 2019; 70: 151-71.

2. Roehlen N, Crouchet E, Baumert TF. Liver Fibrosis: Mechanistic Concepts and Therapeutic Perspectives. Cells. 2020; 9.

3. Moroni F, Dwyer BJ, Graham C, Pass C, Bailey L, Ritchie L, et al. Safety profile of autologous macrophage therapy for liver cirrhosis. Nat Med. 2019; 25: 1560-5.

4. Gill US, Kennedy PTF. The impact of currently licensed therapies on viral and immune responses in chronic hepatitis B: Considerations for future novel therapeutics. J Viral Hepat. 2019; 26: 4-15.

5. Atta HM. Reversibility and heritability of liver fibrosis: Implications for research and therapy. World Journal of Gastroenterology. 2015; 21.

6. Khomich O, Ivanov AV, Bartosch B. Metabolic Hallmarks of Hepatic Stellate Cells in Liver Fibrosis. Cells. 2019; 9.

7. Elias E. Liver failure and liver disease. Hepatology. 2006; 43: S239-42.

8. Puche JE, Saiman Y, Friedman SL. Hepatic Stellate Cells and Liver Fibrosis. Comprehensive Physiology; 2013. p. 1473-92

9. He Y, Huang C, Zhang S-p, Sun X, Long X-r, Li J. The potential of microRNAs in liver fibrosis. Cellular Signalling. 2012; 24: 2268-72.

10. Jiang XP, Ai WB, Wan LY, Zhang YQ, Wu JF. The roles of microRNA families in hepatic fibrosis. Cell Biosci. 2017; 7: 34.

11. Liao X, Zhan W, Tian T, Yu L, Li R, Yang Q. MicroRNA-326 attenuates hepatic stellate cell activation and liver fibrosis by inhibiting TLR4 signaling. Journal of Cellular Biochemistry. 2019.

12. Li J, Dong S, Ye M, Peng G, Luo J, Wang C, et al. MicroRNA-489-3p Represses Hepatic Stellate Cells Activation by Negatively Regulating the JAG1/Notch3 Signaling Pathway. Digestive Diseases and Sciences. 2020.

13. Genz B, Coleman MA, Irvine KM, Kutasovic JR, Miranda M, Gratte FD, et al. Overexpression of miRNA-25-3p inhibits Notch1 signaling and TGF- $\beta$-induced collagen expression in hepatic stellate cells. Scientific Reports. 2019; 9 .

14. Li W-Q, Chen C, Xu M-D, Guo J, Li Y-M, Xia Q-M, et al. The rno-miR-34 family is upregulated and targets ACSL1 in dimethylnitrosamine-induced hepatic fibrosis in rats. FEBS Journal. 2011; 278: 1522-32.

15. Cannell I, Bushell M. Regulation of Myc by miR-34c: A mechanism to prevent genomic instability? Cell Cycle. 2014; 9: 2798-802

16. Naghizadeh S, Mohammadi A, Duijf PHG, Baradaran B, Safarzadeh E, Cho WCS, et al. The role of miR-34 in cancer drug resistance. Journal of Cellular Physiology. 2020.

17. Parkes HA, Preston E, Wilks D, Ballesteros M, Carpenter L, Wood L, et al. Overexpression of acyl-CoA synthetase-1 increases lipid deposition in hepatic (HepG2) cells and rodent liver in vivo. American Journal of Physiology-Endocrinology and Metabolism. 2006; 291: E737-E44.

18. Killion EA, Reeves AR, El Azzouny MA, Yan Q-W, Surujon D, Griffin JD, et al. A role for long-chain acyl-CoA synthetase-4 (ACSL4) in diet-induced phospholipid remodeling and obesity-associated adipocyte dysfunction. Molecular Metabolism. 2018; 9: 43-56.
19. Chen $\mathrm{F}$, Zhang $\mathrm{H}, \mathrm{Li} \mathrm{J}$, Tian $\mathrm{Y}, \mathrm{Xu}$ J, Chen $\mathrm{L}$, et al. Identification of differentially expressed miRNAs in the fatty liver of Landes goose (Anser anser). Scientific Reports. 2017; 7.

20. Friedman SL. Hepatic Stellate Cells: Protean, Multifunctional, and Enigmatic Cells of the Liver. Physiological Reviews. 2008; 88: 125-72

21. Lee TF, Mak KM, Rackovsky O, Lin YL, Kwong AJ, Loke JC, et al. Downregulation of hepatic stellate cell activation by retinol and palmitate mediated by adipose differentiation-related protein (ADRP). J Cell Physiol. 2010; 223: 648-57.

22. Arriazu E, Ge X, Leung T-M, Magdaleno F, Lopategi A, Lu Y, et al. Signalling via the osteopontin and high mobility group box-1 axis drives the fibrogenic response to liver injury. Gut. 2017; 66: 1123-37.

23. Yang L, Dong C, Yang J, Yang L, Chang N, Qi C, et al. MicroRNA-26b-5p Inhibits Mouse Liver Fibrogenesis and Angiogenesis by Targeting PDGF Receptor-Beta. Mol Ther Nucleic Acids. 2019; 16: 206-17.

24. Ju B, Nie Y, Yang X, Wang X, Li F, Wang M, et al. miR-193a/b-3p relieves hepatic fibrosis and restrains proliferation and activation of hepatic stellate cells. J Cell Mol Med. 2019; 23: 3824-32.

25. Murakami Y, Kawada N. MicroRNAs in hepatic pathophysiology. Hepatol Res. 2017; 47: 60-9.

26. Hyun J, Jung Y. MicroRNAs in liver fibrosis: Focusing on the interaction with hedgehog signaling. World Journal of Gastroenterology. 2016; 22.

27. Su Q, Kumar V, Sud N, Mahato RI. MicroRNAs in the pathogenesis and treatment of progressive liver injury in NAFLD and liver fibrosis. Advanced Drug Delivery Reviews. 2018; 129: 54-63.

28. Yan S, Yang XF, Liu HL, Fu N, Ouyang Y, Qing K. Long-chain acyl-CoA synthetase in fatty acid metabolism involved in liver and other diseases: an update. World J Gastroenterol. 2015; 21: 3492-8.

29. Al-Rashed F, Ahmad Z, Iskandar MA, Tuomilehto J, Al-Mulla F, Ahmad R. TNF-alpha Induces a Pro-Inflammatory Phenotypic Shift in Monocytes through ACSL1: Relevance to Metabolic Inflammation. Cell Physiol Biochem. 2019; 52: 397-407.

30. Young PA, Senkal CE, Suchanek AL, Grevengoed TJ, Lin DD, Zhao L, et al. Long-chain acyl-CoA synthetase 1 interacts with key proteins that activate and direct fatty acids into niche hepatic pathways. Journal of Biological Chemistry. 2018; 293: 16724-40.

31. Li LO, Ellis JM, Paich HA, Wang S, Gong N, Altshuller G, et al. Liver-specific Loss of Long Chain Acyl-CoA Synthetase-1 Decreases Triacylglycerol Synthesis and $\beta$-Oxidation and Alters Phospholipid Fatty Acid Composition. Journal of Biological Chemistry. 2009; 284: 27816-26.

32. Lee K-W, Thiyagarajan V, Sie H-W, Cheng M-F, Tsai M-J, Chia Y-C, et al. Synergistic effect of natural compounds on the fatty acid-induced autophagy of activated hepatic stellate cells. The Journal of Nutritional Biochemistry. 2014; 25: 903-13.

33. Moran-Salvador E, Mann J. Epigenetics and Liver Fibrosis. Cell Mol Gastroenterol Hepatol. 2017; 4: 125-34.

34. Quiroga AD, Lehner R. Pharmacological intervention of liver triacylglycerol lipolysis: The good, the bad and the ugly. Biochemical Pharmacology. 2018; 155: 233-41.

35. Anstee QM, Reeves HL, Kotsiliti E, Govaere O, Heikenwalder M. From NASH to HCC: current concepts and future challenges. Nat Rev Gastroenterol Hepatol. 2019; 16: 411-28.

36. Neuman MG, Cohen LB, Malnick S. Familial non-alcoholic steatohepatitis leading to hepatocellular carcinoma. Chemico-Biological Interactions. 2020; 323.

37. Hardy T, Oakley F, Anstee QM, Day CP. Nonalcoholic Fatty Liver Disease: Pathogenesis and Disease Spectrum. Annu Rev Pathol. 2016; 11: 451-96.

38. Li X, Chen $\mathrm{Y}, \mathrm{Wu} \mathrm{S}, \mathrm{He} \mathrm{J}$, Lou $\mathrm{L}$, Ye $\mathrm{W}$, et al. microRNA-34a and microRNA-34c promote the activation of human hepatic stellate cells by targeting peroxisome proliferator-activated receptor $\gamma$. Molecular Medicine Reports. 2015; 11: 1017-24.

39. Yan G, Li X, Peng Y, Long B, Fan Q, Wang Z, et al. The Fatty Acid $\beta$-Oxidation Pathway is Activated by Leucine Deprivation in HepG2 Cells: A Comparative Proteomics Study. Scientific Reports. 2017; 7

40. Li T, Li X, Meng H, Chen L, Meng F. ACSL1 affects Triglyceride Levels through the PPARY Pathway. International Journal of Medical Sciences. 2020; 17: $720-7$.

41. Ding J, Li M, Wan X, Jin X, Chen S, Yu C, et al. Effect of miR-34a in regulating steatosis by targeting PPARalpha expression in nonalcoholic fatty liver disease. Sci Rep. 2015; 5: 13729. 